\title{
Atomic force stiffness imaging: capturing differences in mechanical properties to identify and localize areas of prostate cancer tissue
}

Clara Essmann, Alex Freeman, Vijay M. Pawar, Danail Stoyanov

Clara Essmann, Alex Freeman, Vijay M. Pawar, Danail Stoyanov, "Atomic force stiffness imaging: capturing differences in mechanical properties to identify and localize areas of prostate cancer tissue," Proc. SPIE 10576, Medical Imaging 2018: Image-Guided Procedures, Robotic Interventions, and Modeling, 105761M (13 March 2018); doi: 10.1117/12.2293686 


\title{
Atomic Force Stiffness Imaging: Capturing differences in mechanical properties to identify and localize areas of prostate cancer tissue
}

\author{
Clara Essmann ${ }^{\mathrm{a}}$, Alex Freeman ${ }^{\mathrm{b}}$, Vijay M. Pawar ${ }^{\mathrm{c}}$, \\ Danail Stoyanov ${ }^{a^{*}}$ \\ ${ }^{\mathrm{a}}$ Centre for Medical Image Computing (CMIC) and Dept. Computer Science, \\ University College London, UK \\ ${ }^{\mathrm{b}}$ University College London Hospital, London, UK \\ ${ }^{\mathrm{c}}$ TouchLab, Dept. of Computer Science, University College London, London, UK
}

\begin{abstract}
Prostate cancer is now the most commonly diagnosed cancer in men in western countries. Due to the difficulty for early detection, there are an estimated 10000 deaths a year in the UK from prostate cancer alone; whereby the only curative option is interventional treatment that aims to excise all diseased cells while preserving the neurovascular bundle. To date, several studies have shown that the mechanical properties of cancer cells and tissues i.e. adhesion, stiffness, roughness and viscoelasticity are significantly different from benign cells and regions of tissue that are healthy. Building upon these results, we believe novel methods of imaging the mechanical properties of prostate cancer samples can provide new surgical intervention opportunities beyond what is possible through vision alone. In this paper, we used an Atomic Force Microscope (AFM) to measure the stiffness and topography variations correlating to regions of prostate cancer at the surface of an excised sample at a cellular level. Preliminary results show that by using an AFM we can detect structural differences in non-homogeneous tissue samples, confirming previous results that cancerous tissues appear stiffer than benign areas. Through these results, we aim to develop a stiffness imaging protocol to aid the early detection of prostate cancer, in addition to force sensing surgical tools.
\end{abstract}

Keywords: Prostate cancer, Atomic Force Microscopy Imaging, Tumor Margins

\section{INTRODUCTION}

Stiffness imaging is a method for determining the mechanical and topological characteristics of a material. By providing controlled indentions to the subject matter and analyzing the subsequent contact effects, we can measure underlying material properties i.e. stiffness, adhesion, electrical conductance, viscoelasticity and magnetism at higher resolutions that ordinarily cannot be resolved using traditional optical topographical imaging techniques [1]. Accurate understanding of mechanical properties is becoming increasingly important in medical research when developing synthetic biomaterials for replacement tissues such as teeth, blood vessels, heart valves, cartilage and bones, and skin. Moreover, as the biomechanics of cellular structures play essential roles in signaling during development, and the maintenance of tissue structures in the adult organism, these are also important factors governing tumor growth [2].

The utility of capturing the mechanical properties of cancer cells for localization has been shown in the detection of stiffness changes in comparison to surrounding areas of healthy tissue [3-7]. Analogous to a physician pressing on a tissue to evaluate stiffness by simple palpation, we believe imaging techniques that interpret forces with indentation depth can also be extended for the detection of prostate cancer. In this study, we conduct a feasibility test to evaluate the sensitivity needed to detect structure differences in prostate tissue samples to localize areas of cancer compared with benign areas. This information provides the first step in identifying the force sensing resolution needed to develop a stiffness imaging system for the detection of prostate cancer, in addition to force sensing surgical tools. It also links to 
ongoing studies to develop novel means for imaging tumor margins in the intra-operative setting using a range of optical and molecular imaging techniques $[8,9]$.

To determine the sensitivity range needed to detect variations in prostate cancer samples we used an Atomic Force Microscope (AFM) for its high-resolution measurement capabilities. An AFM is a type of scanning probe microscope (SPM), which uses a fine probe to detect changes to the surface properties of a sample by force-indentation measures [1]. The technique was first developed in the 1980s [10], but capabilities for analyzing soft and sensitive biological materials such as cells and tissues has only emerged with modern cantilever production technologies. Tips assessing the surface can be as soft as $0.01 \mathrm{~N} / \mathrm{m}$ in stiffness and as sharp as $2 \mathrm{~nm}$ in width resulting in much higher magnifications (up to $2 \mathrm{~nm}$ ) than those achieved using a traditional optical microscope and comparable to scanning electron microscopy (SEM). The ability of AFM to perform measurements on unfixed, untreated tissue under physiological conditions at a micro to nano scale, enables the quantification of phenomena as small as van der Waals forces, electrostatic interactions and molecular bonds; providing an opportunity to investigate these nano-characteristics as markers for early stage cancer. Further, as AFM can measure multiple micro- and nano-scale properties in a single test, it presents a useful tool for any field of research that requires simultaneous measurement of topographical features and mechanical properties.

In this study, we used an AFM (Figure 1) to measure the stiffness and topography variations correlating to regions of prostate cancer at the surface of an excised sample at a cellular level. Our results show that we can detect structural differences in non-homogeneous tissue samples, confirming previous results that cancerous tissues appear stiffer than benign areas. By determining this sensitivity range, the purpose of this study is to establish the resolution needed to begin defining a suitable stiffness imaging system for clinical use and to develop a fundamental understanding of the stiffness profile and distribution parameters for prostate cancer across different patients and disease stages.

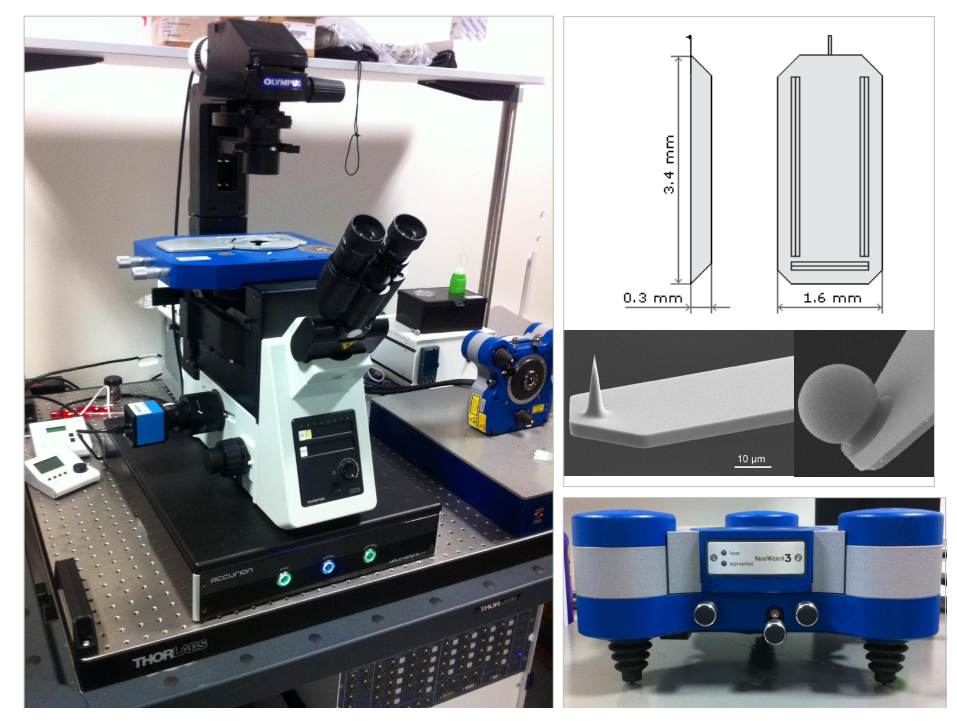

Figure 1: Atomic Force Microscope AFM (NanoWizard3, JPK). Left: AFM set up combining the AFM head and motorized stage (both blue) and the optical microscope for sample visualization. Right: scheme shows dimensions of a standard cantilever (upper panel), two examples of tip shape and size ( $2 \mathrm{~nm}$ (left), $10 \mu \mathrm{m}$ diameter beads (right)) and a close-up of the AFM head (lower panel) 


\section{METHODS}

\subsection{Atomic Force Microscopy}

AFM uses a laser beam deflection system where a laser is reflected from the back of a cantilever and onto a positionsensitive detector (Figure 2). The laser deflection serves as a feedback loop to control tip position and force applied to the sample during the scan. The force is not measured directly, but calculated by measuring the deflection (x) of the cantilever, knowing its stiffness $(\mathrm{k})$. The force $(\mathrm{F})$ can then be calculated using the Hooke's law: $\mathrm{F}=\mathrm{kx}$. The distance a cantilever deflects for a certain change in photodiode voltage depends on the cantilever type and optical path of the AFM and has to be determined each time a cantilever is mounted. For hard surfaces, the cantilever deflection distance is linear to the change in voltage at the photodiode, therefore a glass slide was used to measure the sensitivity (deflection distance per voltage change) prior to each experiment. The cantilever spring constant can be calibrated in different ways including geometric calculations, by using a reference cantilever or by the thermal noise method. Throughout our experiments we used the thermal noise method [11,12]. We used the AFM NanoWizard3 (JPK) and tipless, soft cantilevers with $10 \mu \mathrm{m}$ borsilicate bead attached (0.03-8 N/m; NSC12 $\mu$ Masch) for both force spectroscopy mode (individual force-indentation measures) and quantitative imaging QI mode (combines a raster of force-indentation measures to acquire topographical force maps). Cantilever sensitivity and spring constant were calibrated using the JPK calibration tool [13].

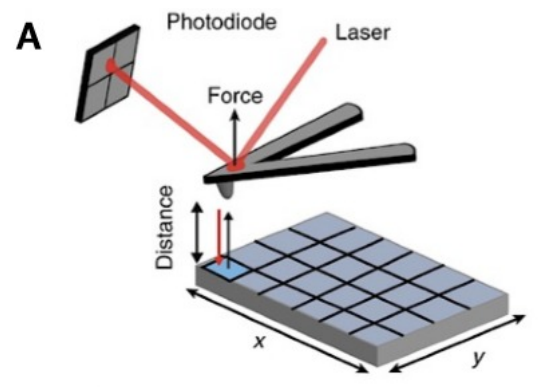

C

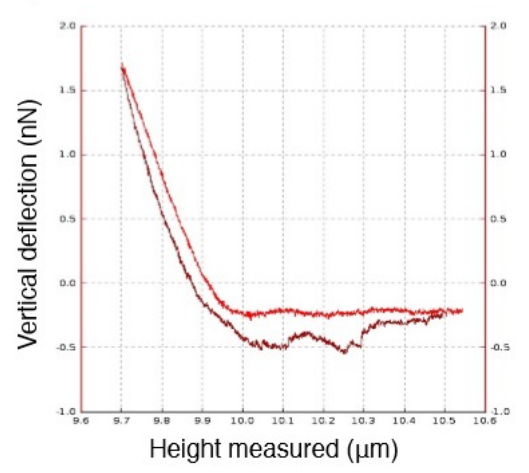

B

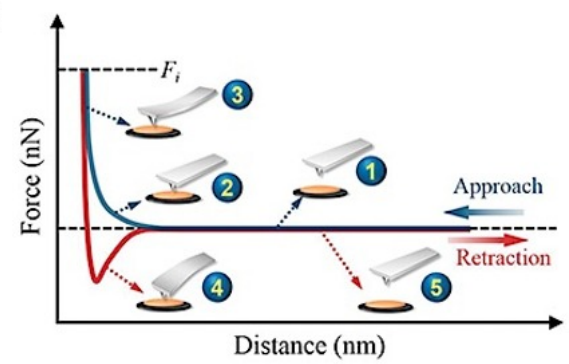

D

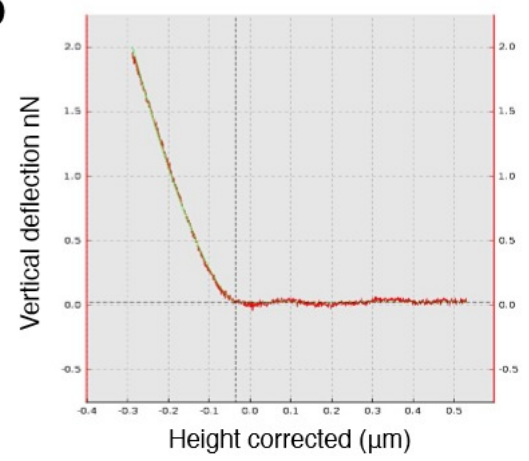

Figure 2: AFM stiffness measures. A Schematic shows operating AFM. A laser reflects from back of cantilever onto a photodiode. Changes in cantilever bending due to material forces divert the laser beam from diode center. Diversion is equivalent with force applied to sample. B Schematic of a typical force-indentation curve acquired by AFM. (1) Approach baseline, no sample contact. (2) Contact point with sample. (3) Maximum indentation force. (4) Retract curve with sample adhesion force. (5) Complete retraction to baseline, no sample contact. C Example force-indentation-curve acquired with the AFM from a prostate tissue sample. D Force-curve example analyzed using the Hertz/Sneddon contact mechanism model to obtain Elastic Modulus of tissue (green curve). 


\subsection{Data analysis}

A typical force measurement consists of an approach and a retract curve, latter usually shows an additional tip sample adhesion force. Initially the height signal in a force-distance curve is the combination of sample deformation and cantilever bending and needs to be corrected for the known cantilever bending to gain the pure sample deformation value termed as tip sample separation. This is an essential step prior to calculating the elastic modulus from the sample and available in the JKP analysis software. The elastic modulus is a value that measures a samples resistance to being deformed temporarily when a stress is applied to it. The stiffer the sample, the higher will be its elastic modulus. The elastic modulus allows comparison of different samples types independent of the way the stress is applied to the sample, unlike stiffness values. For AFM measures, the elastic modulus is widely calculated using the Hertz Model [14] which was further adapted by Sneddon taking indenter shapes into account and is an approximation valid for small indentations only. In our setup we used a small spherical indenter (see Method section 2.1). Using the Hertz-Sneddon contact mechanic model the elastic modulus (green overlay) can be calculated from the approach curve (light red) (Figure 2). AFM data was analysed using JPK analysis software $[12,13]$.

\subsection{Assessment of prostate cancer stiffness}

Cancer tissue is usually identified after fixation of a tissue section followed by histological staining over several days. Using the AFM we are able to measure the stiffness of freshly excised cancerous and non-cancerous tissue already within a few hours after surgery by taking individual force measures over a defined area. The gross location and size of the tumor was estimated via MRI before surgery. After surgical removal the prostate tissue was cut into a slice of $0.5 \mathrm{~cm}$ thickness containing the cancerous area identified via MRI. From this slice samples of about $6 \mathrm{~mm}$ (diameter) x $2 \mathrm{~mm}$ from the cancerous and a similar benign area were collected from the pathology lab and kept on ice in DMEM medium until sample preparation. For measurements, the sample was adhered to a small petri dish using tissue glue and immersed in fresh DMEM. All measurements were obtained at room temperature. The samples can be recovered immediately after the AFM measurements and immersed in tissue fixative. Histological staining proceeds the AFM measurements to confirm the identity and structure of the measured tissue.

\section{RESULTS}

\subsection{Estimation of stiffness for prostate tissue samples}

Several independent force-indentation curves at different locations on the freshly excised tissue sample were obtained for both cancerous and benign samples. The elastic modulus (EM) was calculated from several force-indentation curve measurements obtained for each sample using the Hertz/Sneddon model for contact mechanics (see Methods 2.2). We found an increase in EM in cancerous compared to benign tissue in independent samples (Figure $3 \mathrm{~A}+\mathrm{C}$ ). EM values obtained for the prostate tissue lie within the range of published values $(0.5-8 \mathrm{kPa})$ estimated for other tissues [7]. The distribution of EM values is different across samples sets; showing natural variations between prostate tissues due to factors such as location within the prostate, tumor stage and type. It is therefore essential to collect all available information and to compare benign with cancerous tissue from the same patient. 
A

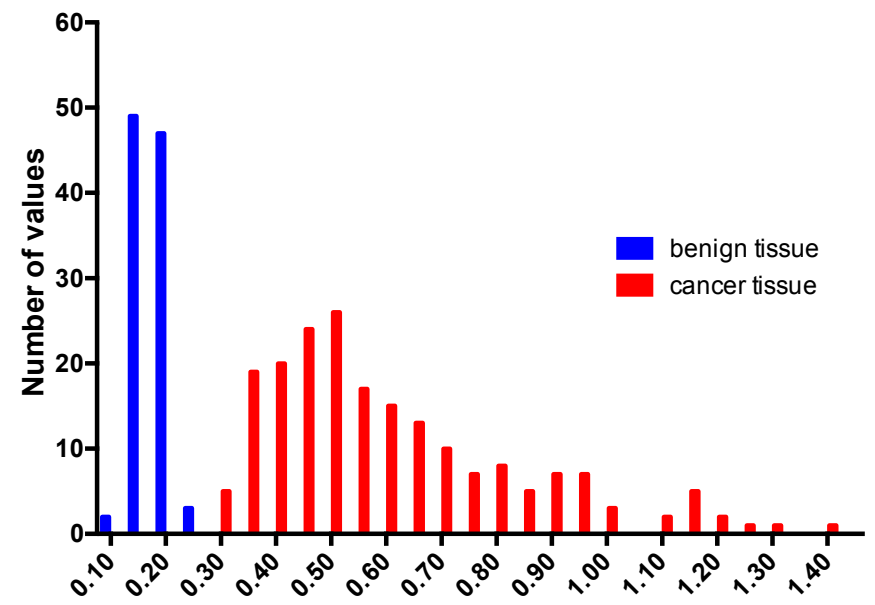

Elastic Modulus (kPa)

C

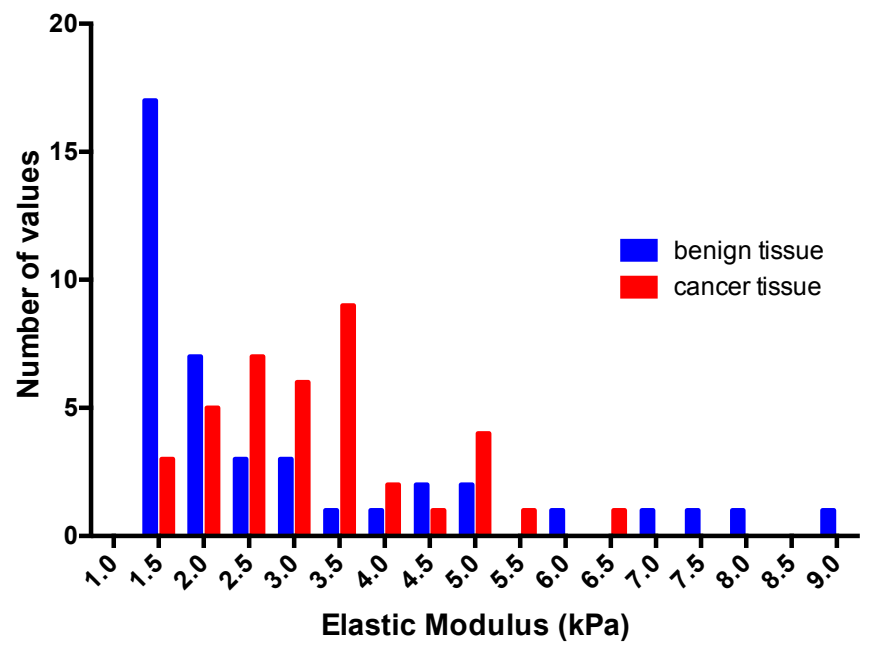

B
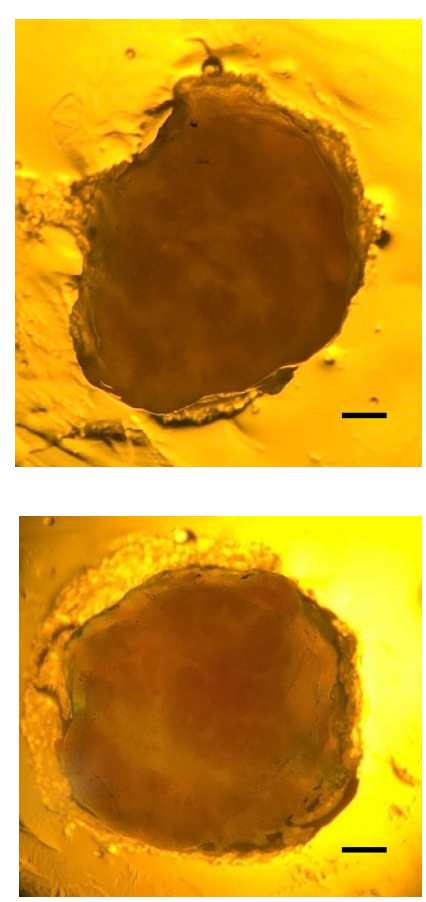

Figure 3: EM of benign and cancerous prostate tissue. A+C Histogram shows frequency distribution of EM obtained from independent force-indentation curves at different location on fresh tissue samples using the Hertz/Sneddon contact mechanics model (A $n>100 ; C \mathrm{n}=41$ ). B Bright field image of fresh benign (top) or cancerous (bottom) tissue sample prepared for AFM. Scale bar $1 \mathrm{~mm}$.

\subsection{D stiffness maps for prostate tissue samples}

Combining force-indentation measures with topographical mapping conducted in a raster-scanning AFM mode (QI) we created 3D-stiffness maps of the two different tissue sections (Figure 4). This application can help identify and visualize structural differences in non-homogeneous tissue samples and confirms our previous results that cancerous tissue appears overall stiffer (up to $8 \mathrm{kPa}$ ) than benign tissue (up to $2.52 \mathrm{kPa}$ ). 

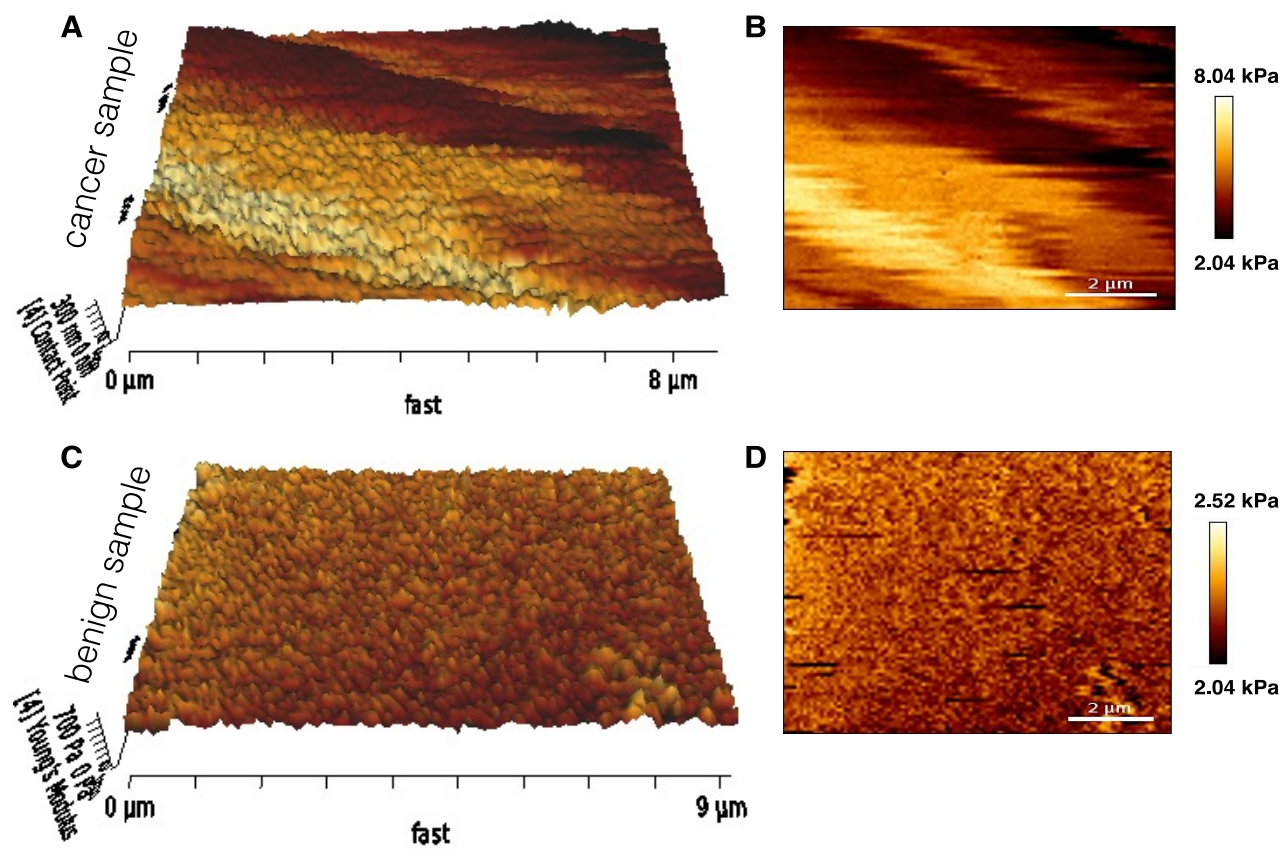

Figure 4: 3D-stiffness map of benign and cancerous prostate tissue. A+C Overlay of topography and Elastic Modulus estimated from force-indentation curves acquired with AFM in QI mode results in 3D-stiffness map of cancerous (A) or benign (C) tissue. B+D 2D stiffness distribution showing color schemed range of Elastic Modulus in cancerous (B) or benign (D) tissue (A+B 128x88pixels, 9.9x8.0 $\mu \mathrm{m}$. C+D 115x88pixels, 9.2x7.0 $\mu \mathrm{m}$ )

Assessing stiffness might be a fast way to identify the refined location of a tumor within a tissue and possibly identify its true margins. The data of this project could help to develop new sensing and surgical systems.

\section{CONCLUSIONS}

This preliminary study investigated the idea of using AFM to delineate healthy and cancerous tissue in excised prostate tissue samples. It is the first study to analyze stiffness changes in human prostate cancer tissue. While we have noted different stiffness map profiles for benign and cancerous tissue, these are limited by the sample size of the current study. Further experiments and data are needed to provide evidence about the domain of values and profile structures we may be able to measure using AFM for this application. In order to achieve deeper understanding of the range and distribution of measurements we may gather, it is necessary to perform additional studies across different patients and pathological presentations. Additional efforts are also needed to develop translation of this capability to clinically practical setups.

\section{ACKNOWLEDGEMENTS}

The work was funded by the EPSRC (EP/M020533/1, EP/P012841/1, EP/N013220/1, EP/N022750/1, EP/N027078/1) and the Wellcome Trust (201080/Z/16/Z). 


\section{REFERENCES}

[1] Jalili, N. \& Laxminarayana, K. A review of atomic force microscopy imaging systems: application to molecular metrology and biological sciences. Mechatronics 14, 907-945 (2004)

[2] P. Janmey and R.T Miller. "Mechanisms of mechanical signaling in development and disease." J. Cell Sci. 124, 9-18 (2011).

[3] S. Park and Y. J. Lee. "AFM-based dual nano-mechanical phenotypes for cancer metastasis", J Bio Phys, 40:413419, (2014)

[4] E. Sariisik et al. "Probing the interaction forces of prostate cancer cells with collagen I and bone marrow derived stem cells on single cell level", Plos One, Vol 8, (2013)

[5] L. Bastastas et al. "AFM nano-mechanics and calcium dynamics of prostate cancer cells with distinct metasatic potential." Biochimica et Biophysica Acta, 1111-1120, (2012)

[6] E. Faria, N. Ma, E. Gazi, P. Gardner, M. Brown, N. Clarke and R. D. Snook. "Measurement of elastic properties of prostate cancer cells using AFM.” Analyst, 133, 1498-1500, (2008)

[7] M. Lekka et al. "Cancer cell detection in tissue sections using AFM." Arch Biochem Biophys, 518(2):151-6, (2012)

[8] K. Vyas, M. Grootendorst, T. Mertzanidou, S. Macholl, D. Stoyanov, S. R. Arridge, D. S. Tuch. "Flexible scintillator autoradiography for tumor margin inspection using ${ }^{18} \mathrm{~F}-\mathrm{FDG}$ ". SPIE Photonics West (2018)

[9] Calvert N., Helo Y., Mertzanidou T., Tuch D. S., Arridge S. R., Stoyanov D. "A simulation study of spectral Cerenkov luminescence imaging for tumour margin estimation" Proceedings of the SPIE, Volume 10137, id. 10137178 pp. (2017)

[10] Binnig, G., Quate, C. F. \& Gerber, C. Atomic force microscope. Phys. Rev. Lett. 56, 930-933 (1986).

[11] Hutter J.L. and Bechhoefer J. Calibration of atomic-force microscope tips. Rev. Sci. Instrum. 64:1868-1873 (1993)

[12] JPK. Summary Notes - A practical guide to AFM force spectroscopy and data analysis. JPK instruments Tech. Summ.

[13] JPK. Summary Notes - Force spectroscopy measurements and processing. JPK instruments Tech. Summ. (May) (2009)

[14] Hertz H. Über die Berührung fester elastischer Körper. Journal für die angewandte Mathematik, 92: 156-171 (1881) 